\title{
NEW DEVICES FOR MEASURING THE CRITICAL CURRENT IN A TAPE AS A FUNCTION OF THE AXIAL AND TRANSVERSE STRAIN, THE MAGNETIC FIELD AND TEMPERATURE
}

\author{
B. ten Haken, A. Godeke and H.H.J ten Kate. \\ Applied Superconductivity Centre, \\ University of Twente, \\ P.O. Box 217, \\ 7500 AE Enschede, the Netherlands.
}

\begin{abstract}
A new set of instruments is developed to induce strain in various directions inside a superconductor. The common goal of these tools is to investigate the influence of the different components in the strain tensor on the current carrying capacity of a $\mathrm{Nb}_{3} \mathrm{Sn}$ tape. This two-dimensional structure is selected as a simple mechanical model system. The first experimental results on the critical current and the critical temperature as a function of the strain in several directions are presented in this paper.
\end{abstract}

\section{INTRODUCTION}

The influence of strain on the electrical properties of superconductors is investigated now for almost three decades. Most of the attention is focused on the A15 type material $\mathrm{Nb}_{3} \mathrm{Sn}$. This brittle superconductor shows a very strong strain dependence in its superconducting properties. Small deformations in the order of $0.2 \%$ can reduce the current carrying capacity significantly. Despite of this extreme mechanical sensibility $\mathrm{Nb}_{3} \mathrm{Sn}$ has become the most important conductor applied in high field magnets in the range from 10 to 20 tesla.

In the investigations described in this paper the simple geometry of a thin $\mathrm{Nb}_{3} \mathrm{Sn}$ tape is selected. In this two dimensional model it is possible to induce a homogeneous strain pattern in different directions inside the sample. In the first two paragraphs the theoretical background and the general considerations for these strain experiments is explained. Then three different strain devices are described and the first experimental results are presented.

\section{THEORY}

For $\mathrm{Nb}_{3} \mathrm{Sn}$ and many other materials the influence of the axial strain on the critical current can usually be described with a scaling law [1]. A fit is made by adding a strain dependence to the upper critical field $\left(\mathrm{B}_{\mathrm{C} 2}\right)$ and by substitution of this parameter into the Kramer rule, which describes the critical current determined by flux pinning.

Manuscript received August 24, 1992.
A fundamental description for the $\mathrm{B}_{\mathrm{C} 2}$ dependence on the full strain tensor is made by D.O. Welch [2]. By assuming a random orientation of the $\mathrm{Nb}_{3} \mathrm{Sn}$ crystals it is found that only two components of the strain tensor will influence the upper critical field. Using experimental data on $\mathrm{Nb}_{3} \mathrm{Sn}$, Welch concluded that the deviatoric strain has a stronger effect on the $\mathrm{B}_{\mathrm{C} 2}$ than the volume strain. With this model one can describe the influence of all possible strains on the critical current, if the complete strain tensor inside the conductor is known.

Several data exist on the behaviour of $\mathrm{Nb}_{3} \mathrm{Sn}$ wires and cables deformed by a transverse load. Sometimes a strong critical current reduction of approximately 20 . $30 \%$ is found in a single wire at a pressure of $100 \mathrm{MPa}$ in a background field of 8 to 10 tesla [3]. Other experiments, on impregnated large cables show a much smaller reduction. Depending on the type of cable a reduction of 3 to $10 \%$ is found under the same conditions [4]. The interpretation of these data is complicated due to the inhomogeneous strain pattern inside these conductors.

\section{A PERFECT STRAIN EXPERIMENT}

Although in the real world there only exits "almost perfect", it is interesting to consider the best possible experiment to investigate the strain dependence of a superconductor. To separate the influence of volume and distortional strain it is necessary to change at least two components of the strain tensor independently. Because the superconducting properties are measured as a macroscopic parameter, usually the voltage drop over a certain distance, it is important that the strain state is uniform in the investigated volume.

Regarding the available conductor geometries there are two possible types of experiments by which one can apply different components of the strain tensor homogeneously: A cylinder geometry (e.g. a superconducting wire) or a flat layer geometry (e.g. a superconducting tape). Thanks to the larger amount of possible strain components which can be applied homogeneously, the layer structure is selected from the two cases above. 
The choice for the layer geometry implies an extra complication. In the case of a superconducting current flowing in combination with a transverse field the fluxpinning process is limiting the critical current density. An eventual anisotropy in the maximum pinning force will introduce a dependence of the critical current on the angular direction of the transverse magnetic field. Such an anisotropic pinning force could be induced by a mechanical strain.

The considerations mentioned above have led to the experiments described in the following paragraphs. The first two strain components selected are: Axial and Transverse strain. $A \mathrm{Nb}_{3} \mathrm{Sn}$ tape is selected as the first sample material. This conductor consists of central tape of niobium where two layers of $12 \mu \mathrm{m} \mathrm{Nb} \mathrm{N}_{3} \mathrm{Sn}$ are formed by reacting the tape in a tin bath. Both the layers are protected by a thin layer of copper. The tape is supplied by Gorizont (G.O.S.) with a width of $5 \mathrm{~mm}$.

\section{THE TWO-COMPONENT STRAIN DEVICE}

The new strain device combines an axial tensile force with a transverse compressive force both acting on a short straight section of the tape conductor. A serious restriction in the design is formed by the $60 \mathrm{~mm}$ bore of the 16 tesla magnet in which the instrument is inserted. The direction of the magnetic field can be changed in two directions by changing the orientation of the sample. Finally the first experimental results on the critical current of the $\mathrm{Nb}_{3} \mathrm{Sn}$ are presented.

\section{A. Experimental setup}

A racetrack like sample geometry is used, with a $25 \mathrm{~mm}$ straight section available for testing (Fig. 1). This shape fits into the $60 \mathrm{~mm}$ magnet bore in all possible directions. The first strain component in the axial direction is introduced by applying a pulling force on the arcs of the racetrack. A mechanical load on the central $20 \mathrm{~mm}$ of the test section, is applied in the transversal direction, with an anvil head on the top of a 1 : 10 lever (Fig. 2a). The maximum capacity of this force is estimated at $10 \mathrm{kN}$.

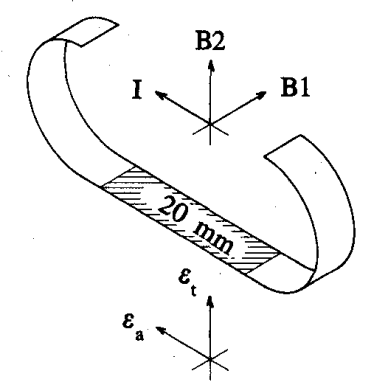

Fig. 1 The racetrack sample geometry.
Both the strain components are adjusted with a wedge and screw construction from outside the cryostat. One measure for the strain in the axial direction is the number of turns of the screw. A more precise determination of the axial strain in the investigated area is made by a strain gage on the tape itself. The force $\left(F_{t}\right)$ in the transverse direction is measured by a strain gage on the lever, which is previously gauged in a separate setup.

Two directions of the magnetic field relative to the sample are investigated. Fig $2 a$ shows the first option with the magnetic field pointing perpendicularly to the transverse force. In Fig. $2 b$ a second option is shown. The sample on the axial strain device is rotated 90 degrees and a second lever system applies a mechanical load transverse to the sample surface and parallel to the magnetic field.

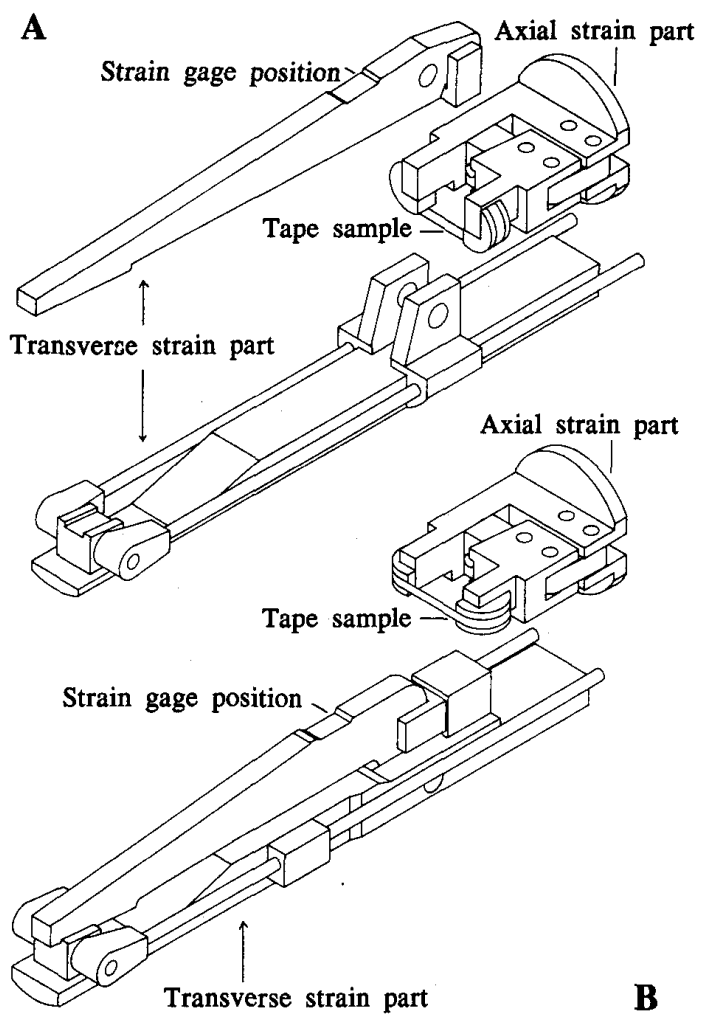

Fig. 2 The two component strain device.

$A$ - first option: $F_{t}$ perpendicular to $B$

$B$ - second option: $F_{t}$ parallel to $B$

\section{B. Critical current reduced by axial strain.}

The first experiments on the axial strain are conducted with the lever for the transverse force replaced by a support block. The proper selection of the direction of the magnetic field and current gives a 
Lorentz force pressing the tape against the support block. The strain is adjusted by small steps of approximately $0.05 \%$. At each step the voltage across the sample was measured as a function of the current, at four different values of the magnetic field.

The critical current is determined with a constant voltage criterion at $1 \cdot 10^{-4} \mathrm{~V} / \mathrm{m}$. The zero of the intrinsic strain is defined by the maximum in the current as found at 10 tesla. The critical current for the tape at this point is 395 amperes, which corresponds to a relatively high current density of $3.3 \cdot 10^{9} \mathrm{~A} / \mathrm{m}^{2}$. The relative reduction of the critical current density as a function of the applied strain is plotted in Fig. 3. A small irreversible reduction (1 to $3 \%$ ) of the critical current is found when an elongation of $0.10 \%$ is applied once. After a deformation of $0.15 \%$ the maximum in the current is found at approximately $10 \%$ lower values.

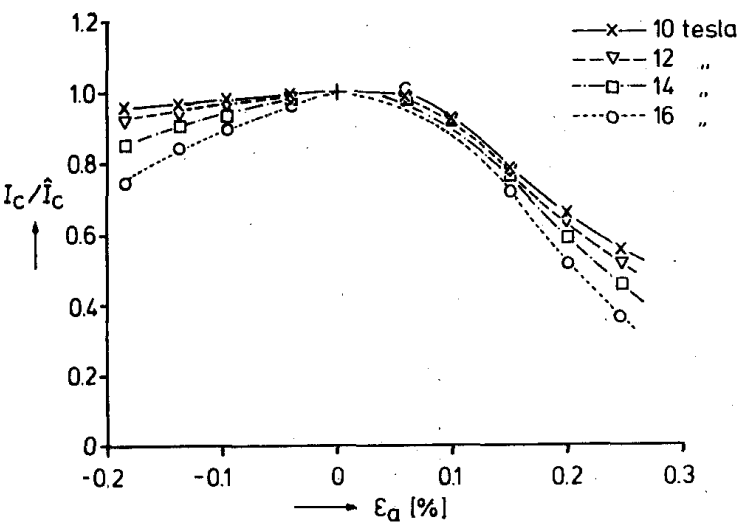

Fig. 3 The scaled current density as a function of the axial strain at $4.2 \mathrm{~K}$.

\section{Critical current reduced by transverse strain.}

The start of the experiments with a strain in the transverse direction is made with the force acting perpendicularly to the magnetic field (The A-option). For this experiment the pressured area is reduced by cutting the tape to a width of $3 \mathrm{~mm}$. The length of the investigated section is $10 \mathrm{~mm}$. The maximum pressure in this configuration is $180 \mathrm{MPa}$. The strain in this case is calculated at $0.13 \%$ by using a Youngs modulus of $137 \mathrm{GPa}$ for the $\mathrm{Nb}_{3} \mathrm{Sn}$ layer [5].

The experimental results on the critical current are obtained with the procedure described above and depicted in Fig. 4. Similar to the axial strain experiment the current reduction is stronger for higher values of the applied magnetic field. It is interesting to compare the results found with the tape to the current degradations found in cases as mentioned above [4]. Approximately the same reduction of $3 \%$ in the critical current at $10 \mathrm{~T}$ and $100 \mathrm{MPa}$ is found in certain types of $\mathrm{Nb}_{3} \mathrm{Sn}$ cables.
Reversibility of the reduction of the critical current is visible only in a very small regime. At a pressure of $40 \mathrm{MPa}(=0.03 \%$ strain) there is already a permanent reduction of the critical current density. This reduction is small $(0.5 \%)$ at 10 tesla but more pronounced $(4.0 \%)$ at 15 tesla. After a pressure of $180 \mathrm{MPa}(=0.13 \%$ strain $)$ is applied this reduction is 8 and $12 \%$ respectively.

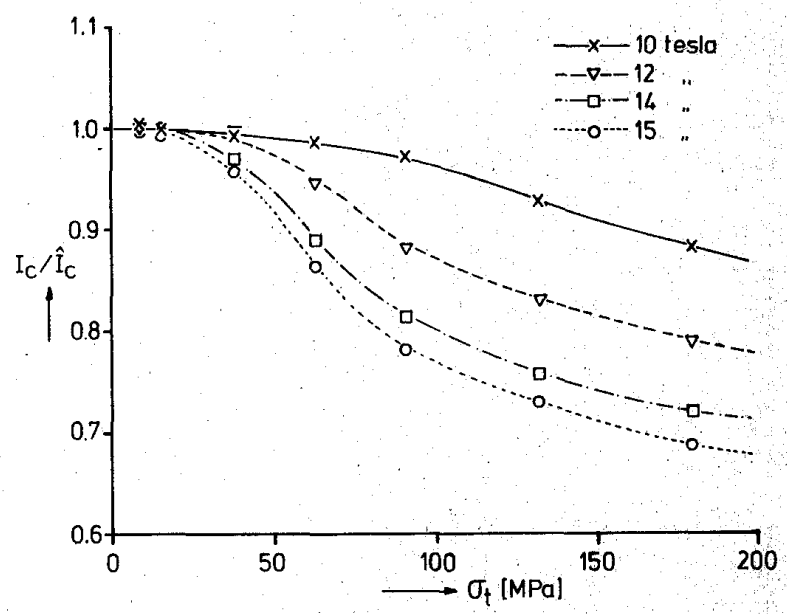

Fig. 4 The relative current density as a function of the transverse pressure at $4.2 \mathrm{~K}$.

The second option of this strain device, with the magnetic field pointing parallel to the transverse force will be investigated in the near future. Also the combination of the two strains will be presented on a next occasion.

\section{TWO AXIAL STRAIN DEVICES}

Although the critical current reduced by axial strain is a well investigated subject, there were good reasons to design also some special instruments for the tape conductor. First the results achieved on the so called U-shaped device are reported. Special attention is paid to the critical current under the influence of a large compressive axial strain. In the second part a compact device to measure the critical temperature as a function of a tensile axial strain is presented.

\section{A. The $U$-shaped strain device}

To induce a homogeneous strain over a wide range from the compressive up to the tensile regime, a special. instrument is developed and described in a previous paper [6]. In this case the sample holder has a U-shaped form which is deformed, together with the super conducting tape, by applying a force on the ends of the $\mathrm{U}$ (Fig. 5). The main advantage of this system is the perfect fixation of the superconductor. 


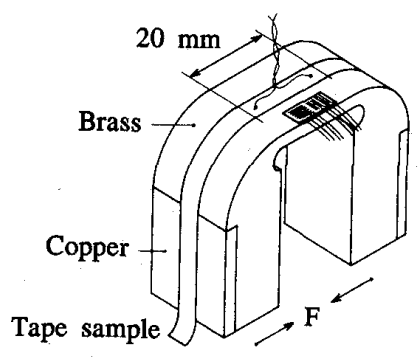

Fig. 5 The U-shaped sample holder.

The critical current in the $\mathrm{Nb}_{3} \mathrm{Sn}$ tape as function of the magnetic field and the applied strain is plotted in Fig. 6. Clearly visible is the extreme reduction (up to $98 \%$ ) of the current at a high magnetic field and a large compressive strain. An interesting effect is the complete reversibility of the critical current over this range from -1.0 to $+0.1 \%$ axial strain.

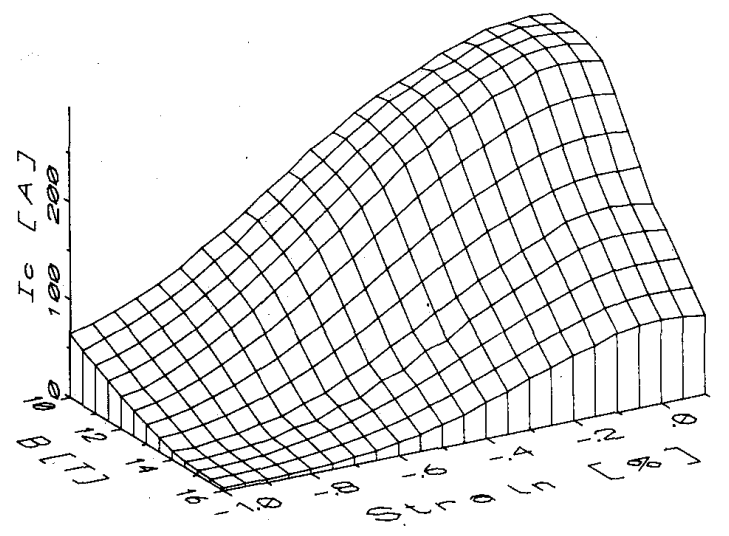

Fig. 6 Critical current as a function of the axial strain and magnetic field at $4.2 \mathrm{~K}$.

\section{B. A strain device with temperature control}

A special axial strain apparatus is developed to investigate the axial strtain effect on the critical temperature. A U-shaped sample is inserted up side down in the $60 \mathrm{~mm}$ magnet bore. The strain device consists of two stainless steel blocks, which can be moved away from each other to elongate the sample. Over this structure there is a small insulation cap made of kapton. The temperature of the entire structure inside the insulation cap is controlled by a heating resistor.

The measured critical temperature of the $\mathrm{Nb}_{3} \mathrm{Sn}$ tape is presented in Fig. 7. The critical temperature is defined at the middle value of the superconducting to normal transition. The zero for the intrinsic strain is defined by the maximum in the critical temperature (at $17.45 \mathrm{~K}$ ). If this strain is exceeded the critical temperature drops rapidly.

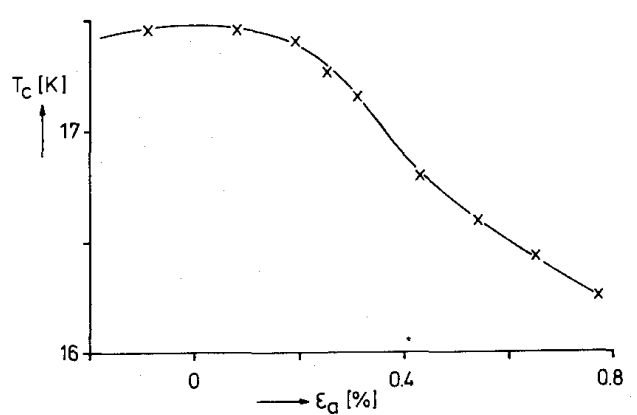

Fig. 7 The critical temperature reduced by axial strain.

\section{CONCLUSIONS}

1- The two dimensional tape structure shows the best perspectives to investigate the strain dependence of the critical parameters of a superconductor.

2- A new set of compact strain instruments that fit into a $60 \mathrm{~mm}$ magnet bore, specially designed for tape conductors, is developed and tested successfully.

3- The first results (on the Gorizont $\mathrm{Nb}_{3} \mathrm{Sn}$ tape) show a comparable reduction on the critical current density due to the axial tensile and the transverse compressive strain.

4- When applying a compressive force in the axial direction a reversible current reduction is found over the entire range up to $1 \%$ deformation. This range is one order larger as the reversible strain range as found in the axial tensile strain and transverse compressive strain case.

\section{REFERENCES}

[1] J.W. Ekin, "Strain effects in superconducting compounds", Adv. in Cryo. Eng. 30, 823-836 (1983).

[2] D.O. Welch, "Alternation of the superconducting properties of A15 compounds and elementary composite superconductors by nonhydrostatic elastic strain", Adv. in Cryo. Eng., 26, 48-56 (1980).

[3] J.W. Ekin, "Effects of transverse compressive stress on the critical current and upper critical field of $\mathrm{Nb}_{3} \mathrm{Sn}$ ", J. Appl. Phys., 62, 4829-4834 (1987).

[4] H.H.J. ten Kate, H.W. Weijers and $H$ van Oort, "Critical-current degradation in $\mathrm{Nb}_{3} \mathrm{Sn}$ cables under high transverse pressure", Proc. ASC-92, Chicago (1992).

[5] G. Rupp. "Parameters affecting prestrain and $\mathrm{B}_{\mathrm{C} 2}$ in multifilamentary $\mathrm{Nb}_{3} \mathrm{Sn}$ conductors", Adv. in Cryo. Eng., 26, 522-529 (1980).

[6] B. ten Haken, A.E. Reitsma and H.H.J. ten Kate,"The reduction of the intrinsic critical current density due to parallel strain in $\mathrm{Nb}_{3} \mathrm{Sn}$ tape like conductors", Proc. ICEC-14, Kiev, (1992), in print. 